\title{
Microglia-associated granule cell death in the normal adult dentate gyrus
}

\author{
Charles E. Ribak • Lee A. Shapiro • \\ Zachary D. Perez $\cdot$ Igor Spigelman
}

Received: 6 August 2009/ Accepted: 11 November 2009/Published online: 21 November 2009

(C) The Author(s) 2009. This article is published with open access at Springerlink.com

\begin{abstract}
Microglial cells are constantly monitoring the central nervous system for sick or dying cells and pathogens. Previous studies showed that the microglial cells in the dentate gyrus have a heterogeneous morphology with multipolar cells in the hilus and fusiform cells apposed to the granule cell layer both at the hilar and at the molecular layer borders. Although previous studies showed that the microglia in the dentate gyrus were not activated, the data in the present study show dying granule cells apposed by Iba1-immunolabeled microglial cell bodies and their processes both at hilar and at molecular layer borders of the granule cell layer. Initially, these Iba1-labeled microglial cells surround individual, intact granule cell bodies. When small openings in the plasma membrane of granule cells are observed, microglial cells are apposed to these openings. When larger openings in the plasma membrane occur at this site of apposition, the granule cells display watery perikaryal cytoplasm, watery nucleoplasm and damaged organelles. Such morphological features are characteristic
\end{abstract}

C. E. Ribak and L. A. Shapiro contributed equally to this paper.

C. E. Ribak $(\bowtie) \cdot$ Z. D. Perez

Department of Anatomy and Neurobiology, School of Medicine, University of California at Irvine, Irvine, CA 92697-1275, USA e-mail: ribak@uci.edu

\section{A. Shapiro}

Department of Surgery and Neurosurgery,

Texas A\&M Health Science Center College of Medicine, Scott \& White Hospital, Central Texas Veterans Health

Care System, Temple, TX 76504, USA

I. Spigelman

Division of Oral Biology and Medicine,

UCLA School of Dentistry,

Los Angeles, CA 90095-1668, USA of neuronal edema. The data also show that following this localized disintegration of the granule cell's plasma membrane, the Ibal-labeled microglial cell body is found within the electron-lucent perikaryal cytoplasm of the granule cell, where it is adjacent to the granule cell's nucleus which is deformed. We propose that granule cells are dying by a novel microglia-associated mechanism that involves lysis of their plasma membranes followed by neuronal edema and nuclear phagocytosis. Based on the morphological evidence, this type of cell death differs from either apoptosis or necrosis.

Keywords Neurodegeneration - Neuronal edema · Neurogenesis · Hippocampus · Membrane lysis

\section{Introduction}

Microglial cells constitute the macrophage population in the normal adult brain and serve as the primary immune component in the central nervous system (CNS). These cells are constantly monitoring the CNS for sick or dying cells, pathogens, and other abnormalities that may require a neuroimmune response (Gehrmann et al. 1995; Nimmerjahn et al. 2005; Davalos et al. 2005). When microglial cells encounter compromised cells, they may act as antigen presenting cells and they can also remove sick or dying cells through the process of phagocytosis (Thanos et al. 1994; Hirt et al. 2000). The distribution of microglial cells has been described in the adult dentate gyrus using immunocytochemical methods for markers, such as Mac-1 and ionized calcium binding adaptor molecule 1 (Iba1) (Wirenfeldt et al. 2003; Jinno et al. 2007; Shapiro et al. 2009). Briefly, they are randomly arranged in the hilus and molecular layer and concentrated on the hilar and molecular layer borders of the 
granule cell layer, where they frequently display a fusiform morphology instead of a multipolar shape (Shapiro et al. 2009). In the hilus, Iba1-labeled cell bodies appear adjacent to capillaries and neuronal somata as well as within the neuropil (Shapiro et al. 2009).

An unresolved issue in adult brain neurogenesis is that the birth of relatively large numbers of new neurons in the hippocampal dentate gyrus does not match the small number of detected dying neurons. Previous studies of granule cell death have demonstrated only small numbers of dying apoptotic cells, and they were exclusively located on the hilar border of the granule cell layer, where neural progenitors reside (Biebl et al. 2000; Ciaroni et al. 2002; Amrein et al. 2004; Kuhn et al. 2005; Ben Abdallah et al. 2007). These studies can only account for a small degree of the granule cell death when compared to the data from several studies of adult neurogenesis that have shown 25-77\% of newborn neurons surviving (Kempermann et al. 1997) from as many as 9,000 cells a day being produced (Cameron and McKay 2001). Although the exact number of surviving newborn granule cells appears to depend on numerous factors, such as age, activity level, and the environment, functional integration of these newborn granule cells has been clearly demonstrated (van Praag et al. 2002; Toni et al. 2007, 2008; Shapiro et al. 2007). Therefore, mature granule cells must be dying to provide space for the newborn neurons to develop and grow their processes. If they were not dying at a rate roughly equal to that of surviving newborn neurons, being produced, the size of the dentate gyrus would continually increase and occupy most of the calvarium.

Mature granule cells are mainly located in the middle and outer thirds of the granule cell layer while newborn neurons migrate from the subgranular zone into the inner third of this layer (Christie and Cameron 2006; OverstreetWadiche and Westbrook 2006; Laplagne et al. 2007). Despite the fact that numerous studies used several markers for degenerating or dying neurons, no studies have described any pathology occurring for granule cells in normal adult brains that are located in the middle or outer portion of the granule cell layer, regions where mainly mature granule cells reside. For example, evidence of neuronal death in this region of the normal dentate gyrus has eluded recognition in studies that used techniques, such as Fluoro-Jade labeling (Schmued and Hopkins, 2000), TUNEL staining (Ciaroni et al. 2002; Kuhn et al. 2005), and immunocytochemistry for markers, such as Caspase-3 and BAX (de Bilbao et al. 1999). Furthermore, the microglia in the dentate gyrus do not generally appear "activated", nor do they appear phagocytic in normal adult animals (Jinno et al. 2007; Shapiro et al. 2008). In fact, exercising mice with their concomitant elevated levels of neurogenesis and survival of newborn neurons in the dentate gyrus also showed microglia in a "resting" state based upon their morphology, surface expression of activity markers, and the expression level of genes associated with neurogenesis (Olah et al. 2009). Therefore, it is plausible to hypothesize that an as yet discovered mechanism is present to remove mature granule cells to accommodate the structural and functional integration of newborn granule cells (van Praag et al. 2002; Kempermann et al. 2003; Zhao et al. 2006; Toni et al. 2007, 2008; Shapiro et al. 2007) without an enlargement of the granule cell layer.

The lack of data on the death of mature granule cells in the adult dentate gyrus raises several important issues; (1) How do they die? (2) Why has their death not been previously observed? (3) Could a novel mechanism be responsible for the death and/or removal of mature granule cells that offsets the functional neurogenesis occurring in the dentate gyrus? The data in the present study provide evidence for a novel mechanism of dentate granule cell death associated with microglial cells. The granule cells appear to be dying by a process termed "microglia-associated granule cell death" that is characterized by openings in the plasma membrane of granule cells, followed by neuronal edema and ending with the phagocytosis of the granule cell's nucleus and damaged organelles. Also, there are no reactive astrocytes adjacent to the pairs of microglial cell and granule cell bodies. This type of neuronal death does not fall under the classical criteria of either apoptosis or necrosis.

\section{Methods}

Animals

Young, adult male Sprague-Dawley rats $(N=5)$ were used for this study. All protocols and experiments were carried out in accordance with the Institutional Animal Care and Use Committee at the University of California, Irvine. The rats were deeply anesthetized with euthasol and transcardial perfusions were carried out using 50-100 ml of $0.9 \%$ sterile saline, followed by $200-250 \mathrm{ml}$ of $4.0 \%$ paraformaldehyde. The brains were allowed to post-fix in the skull for $24 \mathrm{~h}$, after which they were dissected out and placed in $4.0 \%$ paraformaldehyde for $24 \mathrm{~h}$. The brains were then transferred to phosphate-buffered saline (PBS) and $50 \mu \mathrm{m}$ sections were cut using a vibratome.

Iba1 immunocytochemistry

Sections used for immunocytochemistry were incubated in $0.5 \% \mathrm{H}_{2} \mathrm{O}_{2}$ for $30 \mathrm{~min}$, followed by $60 \mathrm{~min}$ in $1 \% \mathrm{H}_{2} \mathrm{O}_{2}$, and then again for $30 \mathrm{~min}$ in $0.5 \% \mathrm{H}_{2} \mathrm{O}_{2}$. Sections were then rinsed with PBS and incubated free-floating in Ibal 
antibody (1:1000, Wako, Osaka, Japan), with 3\% normal goat serum, $0.05 \%$ Triton-X in PBS, for $24 \mathrm{~h}$ rotating at room temperature (RT). The tissue was then rinsed 3 times for $5 \mathrm{~min}$ in PBS and incubated for $1 \mathrm{~h}$ in biotinylated antirabbit IgG (1:200, Vector Labs, Burlingame, CA, USA), rotating at $\mathrm{RT}$. The tissue was then rinsed in PBS 3 times for 5 min each rinse, and incubated for $1 \mathrm{~h}$ in $\mathrm{ABC}$ (Vector Labs) solution, rotating at RT. Following incubation, sections were rinsed with PBS for $20 \mathrm{~min}$ and were developed by incubating in $0.025 \%$ diaminobenzidine (DAB) and $0.002 \%$ hydrogen peroxide, in PBS. The DAB reaction was halted using PBS, followed by three 10 min PBS rinses. In addition, serial sections were reacted for Iba1 immunofluorescence as previously described (Shapiro et al. 2009).

Sections from the hippocampus were processed for electron microscopy using a previously described method (Shapiro and Ribak 2006). Briefly, hippocampal sections containing DAB immunoreaction product within microglial cells were post-fixed in $1 \%$ glutaraldehyde for $1 \mathrm{~h}$, then rinsed in PBS and placed in 1\% osmium tetroxide for 20-60 min, and dehydrated by ethanol and propylene oxide immersion. A flat-embedding procedure was used after which each tissue block was trimmed using a single-edged razor blade under a dissecting microscope. Initially, several semi-thin $(2 \mu \mathrm{m})$ sections containing the dentate gyrus were cut, placed on glass slides, and stained with a toluidine blue solution. Then, a short series of ultrathin (60-80 nm) sections from each block was cut with an ultramicrotome (UltraCut E, Reichert-Jung, Wetzlar, Germany), and sequential sections were collected on mesh and formvarcoated slot grids. The sections were stained with uranyl acetate and lead citrate to enhance contrast. Sections containing the subgranular zone, granule cell layer, and inner molecular layer were examined with a Philips CM-10 transmission electron microscope. Images of Iba1-immunolabeled microglial cells and associated granule cells were captured with a Gatan Ultrascan digital camera.

\section{Trypan blue staining}

Trypan blue staining of hippocampal slices obtained from an additional group of young adult rats $(N=5)$ was conducted following a method described by Colwell et al. (1996). Briefly, $400 \mu \mathrm{m}$ hippocampal slices from young adult rats were placed into oxygenated, artificial cerebrospinal fluid (ACSF) for $1 \mathrm{~h}$ at $20^{\circ} \mathrm{C}$, and then exposed to $0.4 \%$ trypan blue in ACSF for $10 \mathrm{~min}$. The trypan blue was rinsed from the slices, and they were then fixed in $4 \%$ paraformaldehyde in $0.1 \mathrm{M}$ phosphate buffer for $24 \mathrm{~h}$ at $4^{\circ} \mathrm{C}$. Sections $(50 \mu \mathrm{m})$ were cut from these slices and examined with brightfield optics. Sections containing trypan blue-labeled cells within the granule cell layer were immunolabeled for Iba1 as previously described. Only sections from the middle third of the hippocampal slices were included in the double-labeling analysis with Iba1 to decrease the likelihood of including those granule cells which may have been mechanically compromised near the slice surface during the preparation of the slices.

\section{Results}

Iba1-immunolabeled cells were examined using light and confocal microscopy to determine their distribution in the rat dentate gyrus. Consistent with previous reports of the distribution of microglial cells using this and other markers (Dalmau et al. 1998; Moga et al. 2005, Shapiro et al. 2009), Iba1-labeled microglial cells were found throughout the hilus, at the border of the hilus and granule cell layer and in the molecular layer (Fig. 1). The spacing between the Iba1-labeled cell bodies in the hilus was relatively constant, as previously shown (Shapiro et al. 2009; Jinno et al. 2007). In contrast, several Iba1-labeled cells were grouped closer together at the border between the hilus and the granule cell layer, i.e., the subgranular zone (Fig. 1). The Iba1-labeled cell bodies at this location were fusiform

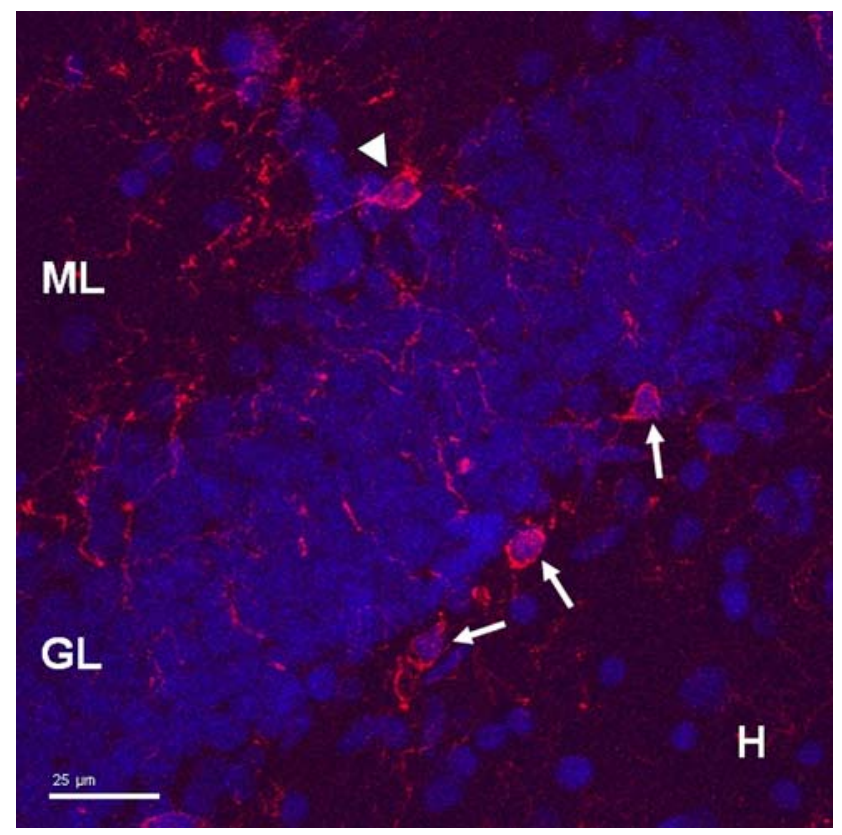

Fig. 1 Distribution of Iba1-labeled cell bodies that are associated with the granule cell layer. DAPI 405 stain (blue) was used for all nuclei, including those in the granule cell layer (GL), and Alexafluor 555 antibody was used to indicate the Iba1-immunolabeled microglial cells (red). Three Iba1-labeled cell bodies (arrows) are found at the border between the GL and the hilus $(\mathrm{H})$, and another Ibal-labeled cell body (arrowhead) is located on the molecular layer (ML) border with the GL. Notice the thin, short processes emanating from these Iba1-labeled cells as well as processes found within the GL (red threads). Scale bar $25 \mu \mathrm{m}$ 
(see Fig. 1) as compared to those in the deep hilus, where they displayed round cell bodies (not shown). The molecular layer had a relatively even distribution of Iba1-labeled microglial cells. However, several were found at the border with the granule cell layer, where they had processes extending into the granule cell layer (Figs. 1, 2). Iba1labeled cells in the granule cell layer were relatively infrequent. This distribution was similar to that reported for the dentate gyrus of mice (Wirenfeldt et al. 2003; Jinno et al. 2007). It should be noted that the Iba1-labeled cells found at the hilar and molecular layer borders of the granule cell layer apposed the cell bodies of granule cells (Fig. 1).

Semi-thin, $2 \mu \mathrm{m}$ sections that were counterstained with toluidine blue were used to analyze the Iba1-labeled microglial cells and the granule cell bodies that they apposed (Figs. 2, 3) as the cells were easily distinguished in these preparations. The distribution of Iba1-labeled microglial cells in these $2 \mu \mathrm{m}$ sections was the same as that described above. Thus, most of the Iba1-labeled cells that were associated with the granule cell layer were found both at the hilar and at the molecular layer borders while few were found within this layer (Figs. 2, 3). Individual Iba1labeled microglial cells were apposed to the cell body of granule cells (Fig. 2). In these instances, the cell body of the Iba1-labeled cell formed a curve around the granule cell body, while its Iba1-labeled processes (sometimes with kinks) also curved around the same granule cell body (Fig. 2). Commonly, such granule cells and their apical dendrites were hypertrophied (Fig. 3d). Also, the nuclei of these granule cells that were mostly enveloped by the Iba1labeled microglial cells were not clearly visible, while adjacent granule cells showed distinct nuclei. In other instances, the Iba1-labeled cell body was apposed to the nucleus of a granule cell with no apparent perikaryal cytoplasm between them (Figs. 2, 3). Other Iba1-labeled microglial cells did not have any processes but instead appeared to be within a void in the granule cell layer (Fig. 3). Such voids did not appear to be capillaries because there were no endothelial cells lining the void (Fig. 3). Therefore, the Iba1-labeled cells exhibited different morphologies in the granule cell layer.

Electron microscopy confirmed the apposition of Iba1labeled microglial cells to granule cell bodies and demonstrated greater detail than the light microscopy (Fig. 4). As shown previously (Shapiro et al. 2009), the immunoreaction product for Iba1 was found within the perikaryal cytoplasm and processes of microglial cells (Fig. 4b, c). The labeling in the cell bodies of Iba1-labeled microglial cells could be distinguished from the distinctive chromatin pattern of their nuclei, where heterochromatin is adjacent to the nuclear envelope while the center of the nuclei had lighter chromatin (Fig. 4b). Iba1-labeled microglial cells apposed granule cells at both the hilar and molecular layer borders of the granule cell layer. The Iba1-labeled processes of these microglial cells appeared as small profiles randomly distributed around the periphery of the granule cell body (Figs. 4a, 5a). The Iba1-labeled cell body and some of its processes apposed the granule cell body while other Iba1-labeled processes were separated from the granule cell body by profiles of dendrites and axon terminals, some of which formed axosomatic synapses (Fig. 4c). However, other granule cells apposed by Iba1-labeled microglial cells had watery cytoplasm at the site of apposition to either a process or cell body of the Iba1-labeled cell (Fig. 5). This change in the appearance of the cytoplasm was not an artifact of fixation because the remainder of the cell body appeared normal and the adjacent tissue

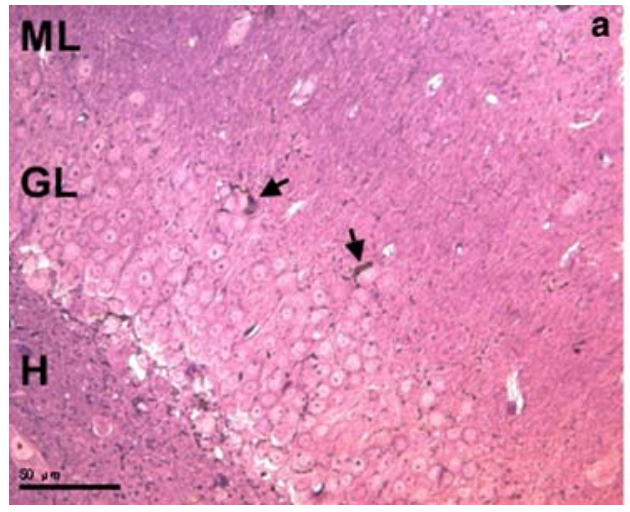

Fig. 2 Semi-thin $2 \mu \mathrm{m}$ section from a normal rat dentate gyrus to show Iba1-labeled cells and processes apposed to granule cell bodies. a A low magnification of two Iba1-labeled cell bodies (arrows) at the molecular layer (ML) border with the granule cell layer (GL); b An enlargement of these two Iba1-labeled cells (arrows) that are each cradling a cell body of a granule cell (blue arrowheads). Not only

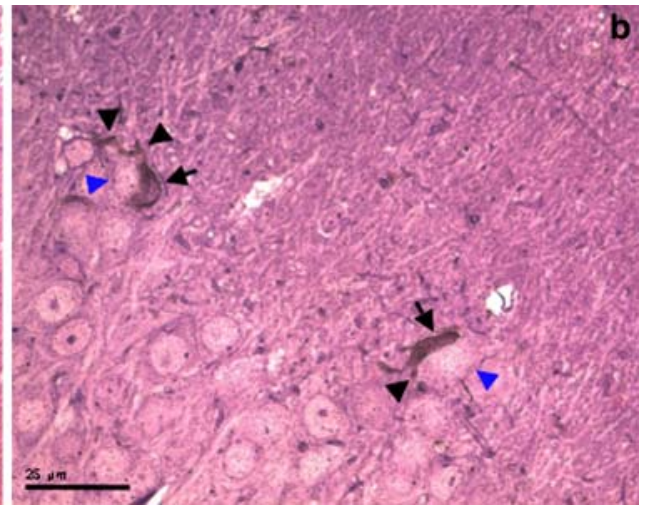

does the cell body of each Iba1-labeled cell form a curve around the granule cell body but their processes (black arrowheads) also curve around these same granule cell bodies. The process of the Iba1labeled microglial cell body on the left has kinks in it, while the Iba1labeled cell on the right abuts against a non-distinct nucleus of the granule cell it apposes. Scale bars $50 \mu \mathrm{m}$ for $\mathbf{a}$, and $25 \mu \mathrm{m}$ for $\mathbf{b}$ 


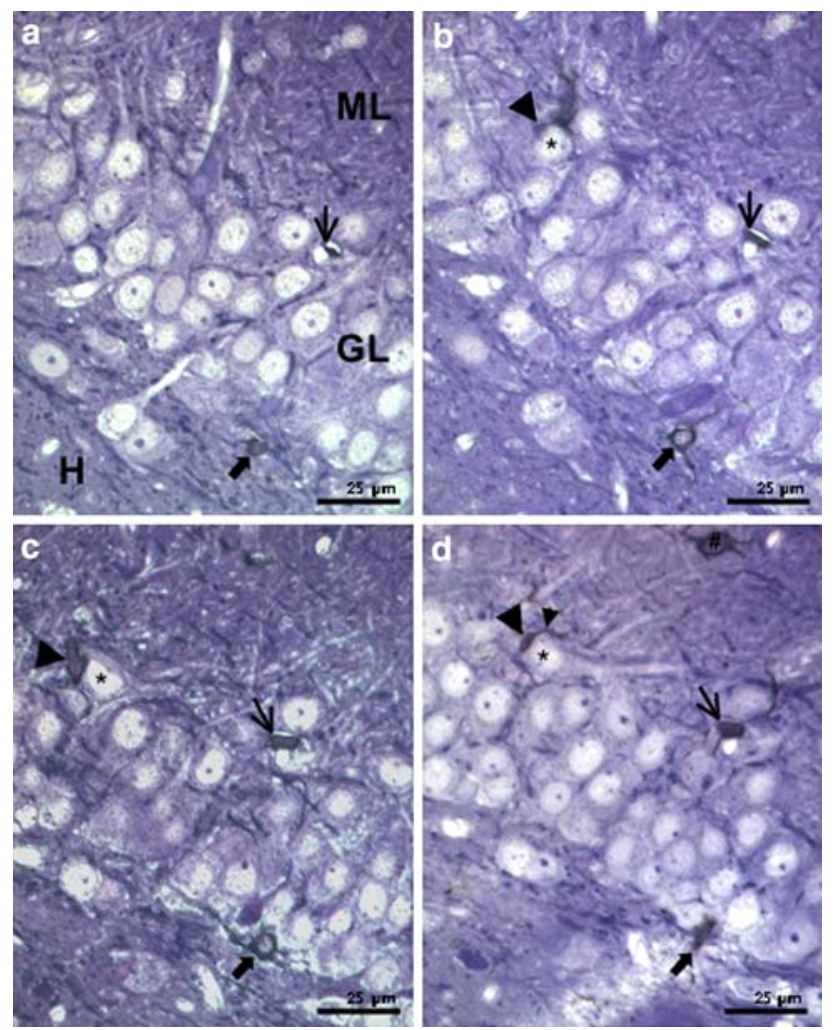

Fig. 3 Serial $2 \mu \mathrm{m}$ sections of three Iba1-labeled cells and processes associated with the granule cell layer (GL). One of the Iba1-labeled cells (arrows, Panels a-d) is located within the outer third of the GL and appears to be within the shell of a former granule cell because of the presence of a clear space around it that is not lined by endothelial cells for a capillary. Another Iba1-labeled cell body (arrowheads, Panels b-d) is located adjacent to the nucleus (asterisk, Panels b-d) of a granule cell at the molecular layer border. The proximal apical dendrite arising from this granule cell that is oriented at a 3 o'clock position appears hypertrophied. Note that a process (small arrow in Panel d) from this same Iba1-labeled microglial cell partially surrounds the apposed granule cell body. The third Iba1-labeled cell (bold arrow, Panels a-d) is found at the border between the hilus $(\mathrm{H})$ and GL and has several processes arising from it. Another Iba1labeled cell body (\#, Panel d) is found in the molecular layer (ML). Scale bar $25 \mu \mathrm{m}$ in $\mathbf{a}-\mathbf{d}$

displayed well-fixed profiles of granule cells, dendrites, axons, and astrocytes (Fig. 5b). It needs to be emphasized that an actual disruption in the integrity of the granule cell's plasma membrane was observed at the site apposed by the Iba1-labeled microglial processes and cell bodies so that the cytoplasm of the granule cell was confluent with the extracellular space (Fig. 5b). Other granule cell bodies that were apposed by Iba1-labeled microglial cell bodies had a greater extent of their plasma membrane disrupted at sites of apposition (Fig. 6). In these cases where many openings were found along a larger extent of apposed plasma membrane (Fig. 6b), the entire cell body of these granule cells appeared watery, including its nucleoplasm and perikaryal cytoplasm (Fig. 6a; cf., the normal appearing granule cell bodies in its vicinity). In addition, many cellular organelles in the watery perikaryal cytoplasm of such granule cells were damaged (Fig. 6a). For example, cisternae of the endoplasmic reticulum were dilated, and mitochondria were swollen and appeared larger than those in adjacent granule cells (Fig. 6a). The watery perikaryal cytoplasm, watery nucleoplasm, and morphological changes to certain organelles are consistent with the hypothesis that extracellular fluid has entered the granule cell through perforations in its somatic plasma membrane.

Electron microscopy showed an additional striking relationship for Iba1-labeled microglial cells and granule cell bodies: the microglial cell bodies were found within the granule cell bodies (Figs. 7a, 8a). Examples of this relationship were found both at the hilar and at the molecular layer borders of the granule cell layer (Fig. 7a). The Iba1-labeled microglial cells either apposed the nucleus of granule cells with some organelles intervening (Fig. 7b) or indented the typically round/oval nucleus where it appeared to disrupt the nuclear envelope (Fig. 8a, c). It should be noted that the granule cells with microglial cells adjacent to their nuclei had the same features as the granule cells with disrupted plasma membranes; i.e., watery perikaryal cytoplasm, watery nucleoplasm, and compromised organelles (cf., Figs. 6a, 8b). Furthermore, when the granule cell body had its nucleus indented by a microglial cell body, organelles were collapsed and condensed (Fig. 8b). Another unusual aspect for these Iba1labeled microglial cell bodies within granule cell bodies was that they lacked processes in the plane of the section. Also, there were no reactive astrocytes observed adjacent to the pairs of microglial cells and granule cell bodies. Instead, normal looking neuropil with axonal and dendritic profiles are adjacent to these cells (Figs. 7, 8).

To test the integrity of dentate granule cells and their plasma membranes, the trypan blue exclusion method was used on hippocampal slices. Intact neurons normally exclude dyes, such as trypan blue, whereas compromised cells in brain slices are labeled with this dye (Colwell et al. 1996). The data from this experiment showed several trypan blue-labeled cells in the granule cell layer from sections obtained from the center of hippocampal slices. Iba1-labeled microglial cells were observed to appose these trypan blue cells (Fig. 9).

\section{Discussion}

The data from this study address a novel mechanism regarding the death of granule cells in the normal adult dentate gyrus. This discussion is divided into sections describing the novel pathway for cell death, the location of 

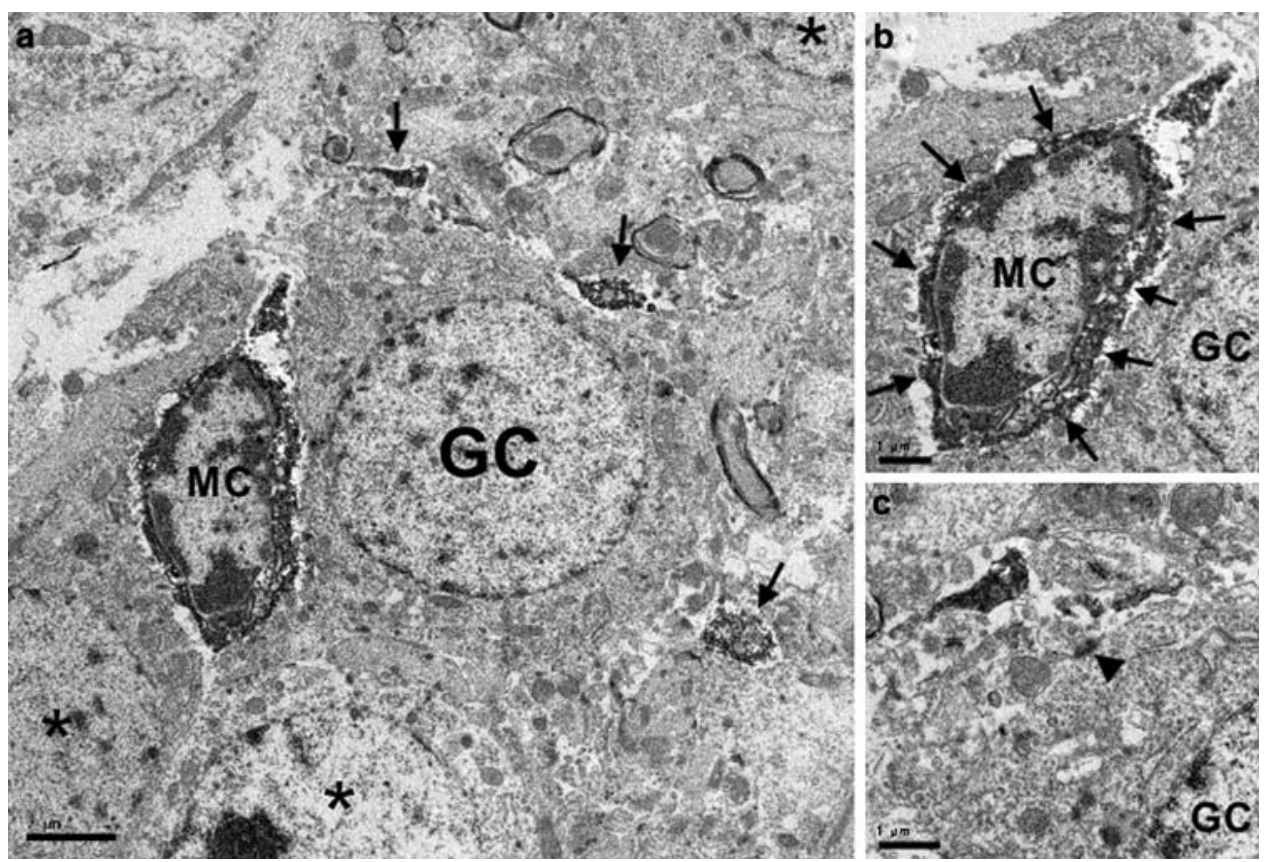

Fig. 4 Electron micrographs of an Iba1-labeled microglial cell with processes surrounding a granule cell at the granule cell/molecular layer border. a The microglial cell body (MC) and several processes (arrows) surrounding a granule cell body (GC). Nearby granule cells (asterisks) are not surrounded by this Iba1-labeled microglial cell and its processes. b An enlargement of the apposition between the microglial cell (MC) and granule cell (GC) found in $\mathbf{a}$. Note the dense
Iba1-immunoreaction product (arrows) contained within the perikaryal cytoplasm of the microglial cell. The pattern of chromatin in this microglial cell is similar to that previously described (Shapiro et al. 2009). c A higher magnification of the axosomatic synapse (arrowhead) on the granule cell (GC) found in a. Scale bars $2 \mu \mathrm{m}$ in a, and $1 \mu \mathrm{m}$ in $\mathbf{b}$ and $\mathbf{c}$

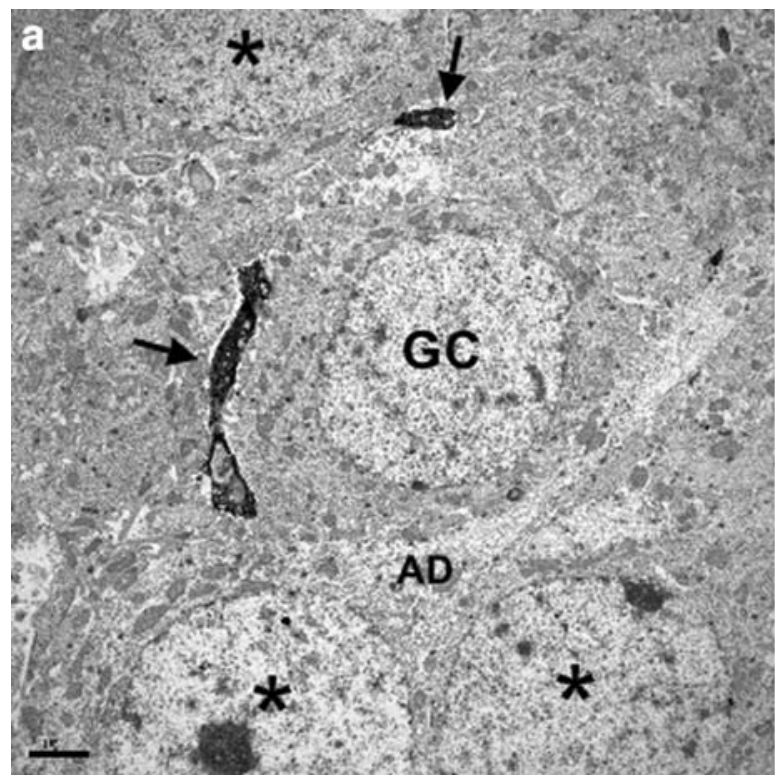

Fig. 5 Electron micrographs of Iba1-labeled processes partially surrounding a granule cell at the granule cell/molecular layer border. a The Iba1-labeled processes (arrows) apposed to the plasma membrane of an adjacent granule cell (GC). Other granule cells (asterisks) are not apposed by these processes and one of them shows an apical dendrite (AD) extending into the molecular layer. b A region of the granule cell body that is apposed by the top Iba1-labeled

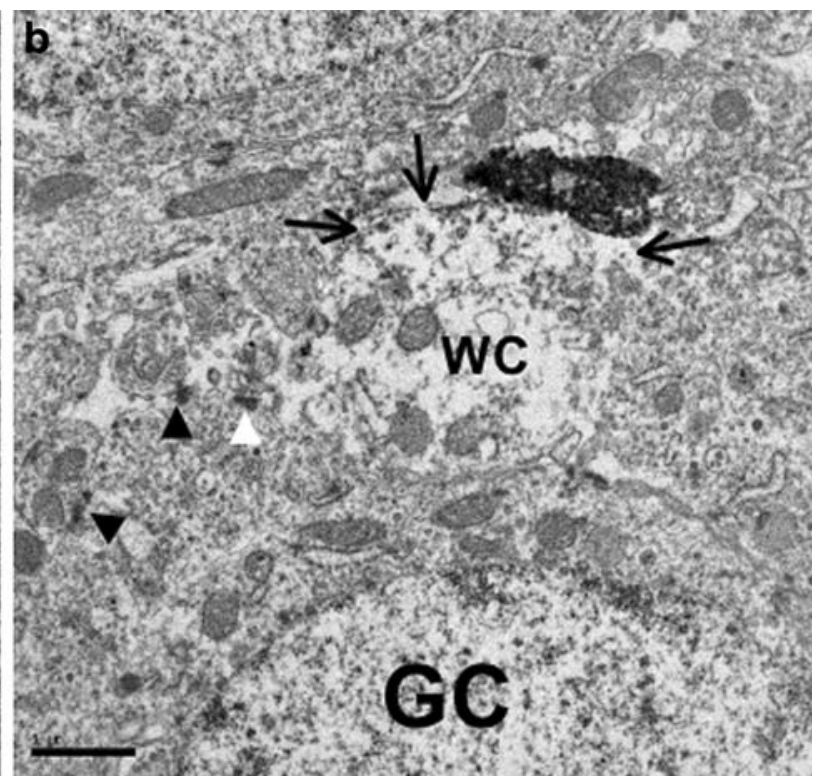

process in a. Note that this part of the cell body has its plasma membrane disrupted in a few places (arrows) and its perikaryal cytoplasm is watery (WC) at this location. Two intact (black arrowheads) axosomatic synapses and a degenerating one (white arrowhead) are found on the surface of this granule cell body. Note that the tissue surrounding the immunolabeled processes and this cell is well fixed. Scale bars $2 \mu \mathrm{m}$ in $\mathbf{a}$, and $1 \mu \mathrm{m}$ in $\mathbf{b}$ 


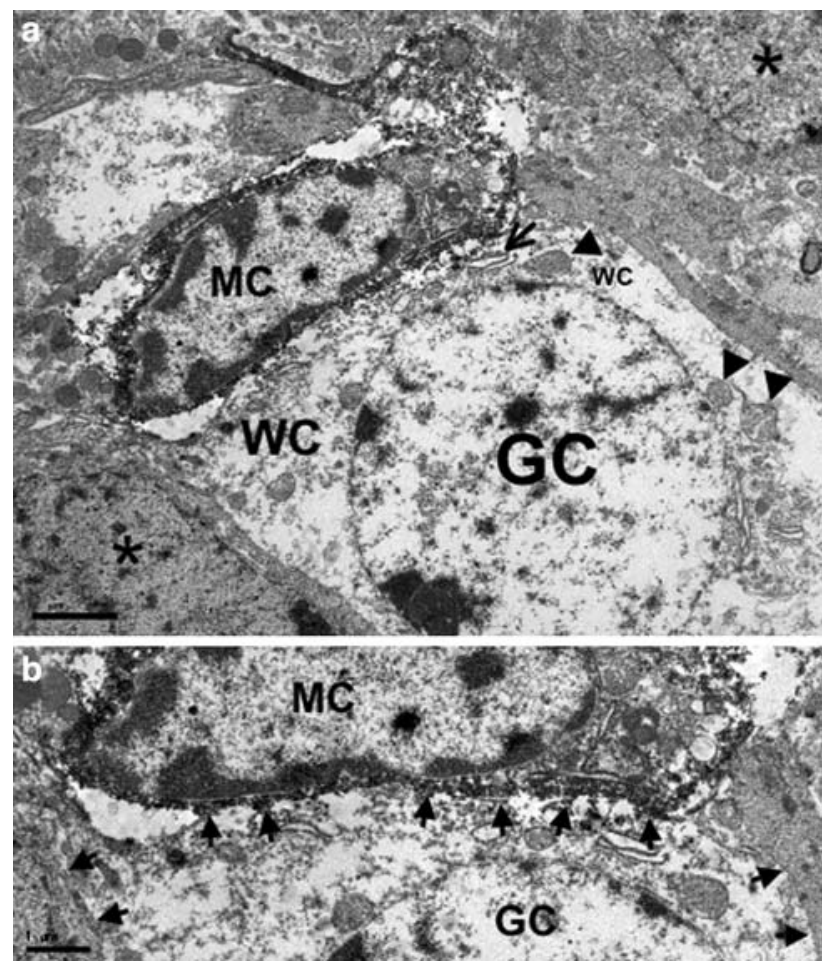

Fig. 6 Electron micrographs of an Iba1-labeled microglial cell apposed to a granule cell body with extensive watery cytoplasm. a A microglial cell body (MC) directly in contact with the plasma membrane of a granule cell body (GC). Watery perikaryal cytoplasm (WC) is found throughout this cell body as well as watery nucleoplasm. Compare with the two adjacent granule cell bodies (asterisks). Note that this watery cytoplasm (WC) contains swollen mitochondria (arrowheads) and cisternae of endoplasm reticulum (arrow). In $\mathbf{b}$, the plasma membrane of the granule cell (GC) is extensively disrupted where it is apposed by the microglial cell body (MC). Arrows indicate the sites of intact plasma membranes. Scale bars $2 \mu \mathrm{m}$ in $\mathbf{a}$, and $1 \mu \mathrm{m}$ in $\mathbf{b}$

the dying granule cells, the morphology of the microglial cells associated with the dying granule cells, and technical considerations.

\section{Novel pathway for neuronal death}

This study of Iba1-labeled microglial cells and the granule cells associated with them provides morphological data about a novel type of neuronal death that occurs in the granule cell layer of the normal adult dentate gyrus. The data indicate that microglial cells are involved in the death of dentate granule cells by a phenomenon consisting of four morphological stages (see drawings in Fig. 10). Initially, the microglial cell body and processes surround a granule cell body (Fig. 10a). At the next stage, the plasma membrane of the granule cell displays openings that are apposed by the microglial cell (Fig. 10b). Following this stage of initial plasma membrane disruption, the granule cell's plasma membrane shows more perforations, its cell body is hypertrophied (Fig. 10c) and displays electronlucent perikaryal cytoplasm, watery nucleoplasm, and damaged organelles. Thus, we suggest that the lysis of the apposed granule cell's plasma membrane results in extracellular fluid flooding into these granule cells through the disrupted plasma membrane, resulting in neuronal edema. In the final stage, we suggest that the highly perforated plasma membrane of the granule cell disintegrates at the site of microglial cell apposition causing the microglial cell body to enter the shell of the granule cell body where it apposes the granule cell's nucleus (Fig. 10d). It remains to be determined whether this nucleus only deforms or degenerates. Nevertheless, these data provide morphological evidence for a novel pathway for a dentate granule cell's demise via plasma membrane lysis and subsequent neuronal edema in the normal adult brain. We have termed this process, "microglia-associated granule cell death" and propose that this loss of plasma membrane integrity is irreversible.

It should be emphasized that the pathway for cell death described in this paper is unique and differs both from apoptosis and from necrosis. A previous electron microscopic study of dying granule cells from epileptic rats has shown that they undergo apoptosis while hilar neurons undergo necrosis (Sloviter et al. 1996). The dying granule cells in the present study that are associated with Iba1labeled microglial cells do not display the distinct morphological features of either of these well-characterized cell deaths. Although another electron microscopic study of apoptosis and necrosis suggests a morphological continuum from one to the other (Portera-Cailliau et al. 1997), it should be emphasized that neither begins with the perforation of the plasma membrane. Therefore, it appears that many granule cells in the adult dentate gyrus may die via a novel pathway that has eluded description until now.

Another interesting aspect of this microglia-associated granule cell death is that the target for the microglial cell is the cell body of the dentate granule cell and not its apical dendrite. The apical dendrites of granule cells apposed by microglial cells hypertrophied at the light microscopic level (see Fig. 3d), but were not targeted by microglial cells. In contrast, Ackman et al. (2006) showed that microglia fused with the apical dendrites of neocortical pyramidal cells that were infected with retrovirus. Future studies may help to understand why microglia target different neuronal regions for neurons from different brain regions.

Distribution of microglia-associated dying granule cells

Previous studies using markers that are typically used to detect cell death by necrosis have failed to detect substantial numbers of dying granule cells in the normal adult 


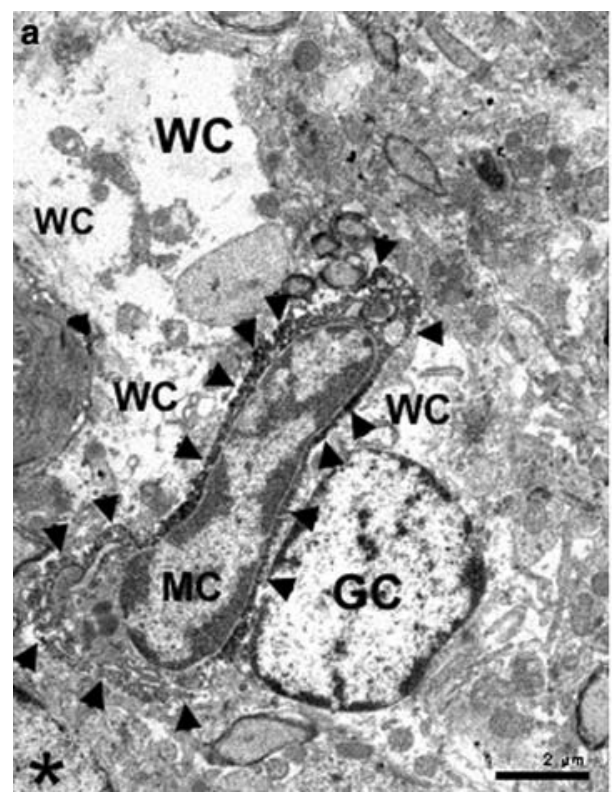

Fig. 7 Electron micrographs of an Iba1-labeled microglial cell body found within a granule cell body at the hilar border of the granule cell layer. a The microglial cell body (MC) displays Iba1-immunoreaction product (arrows) in its perikaryal cytoplasm. This microglial cell lies within the perikaryal cytoplasm of a granule cell (GC). The perikaryal cytoplasm of this granule cell is watery (WC) and is found on both sides of this microglial cell. Compare with the cytoplasm and

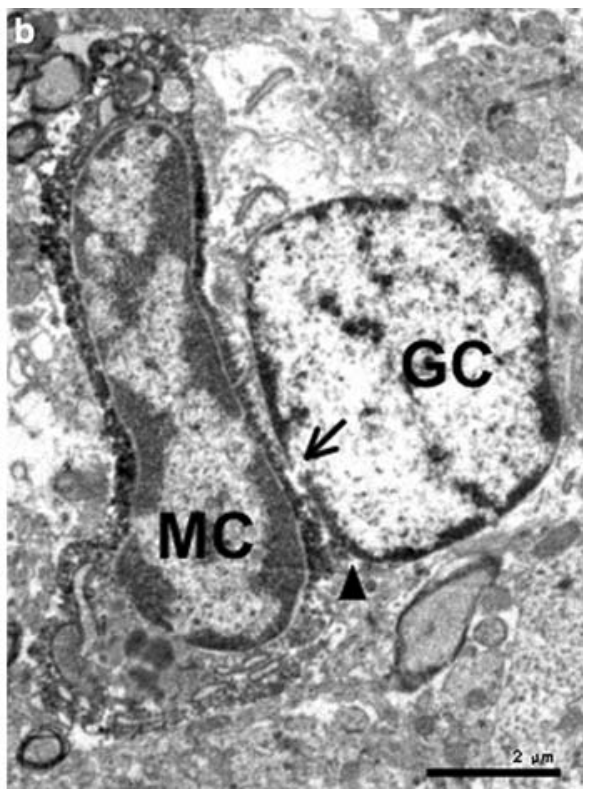

nucleoplasm of a nearby granule cell (asterisk). b An enlargement of the two cell bodies to show that the granule cell's plasma membrane is missing between its nucleus and the microglial cell (MC). Note that the nuclear envelope of this granule cell's nucleus (GC) appears disrupted at one point, (arrow), bringing the nuclear contents in contact with the microglial cell. Scale bars $2 \mu \mathrm{m}$ in $\mathbf{a}$ and $\mathbf{b}$
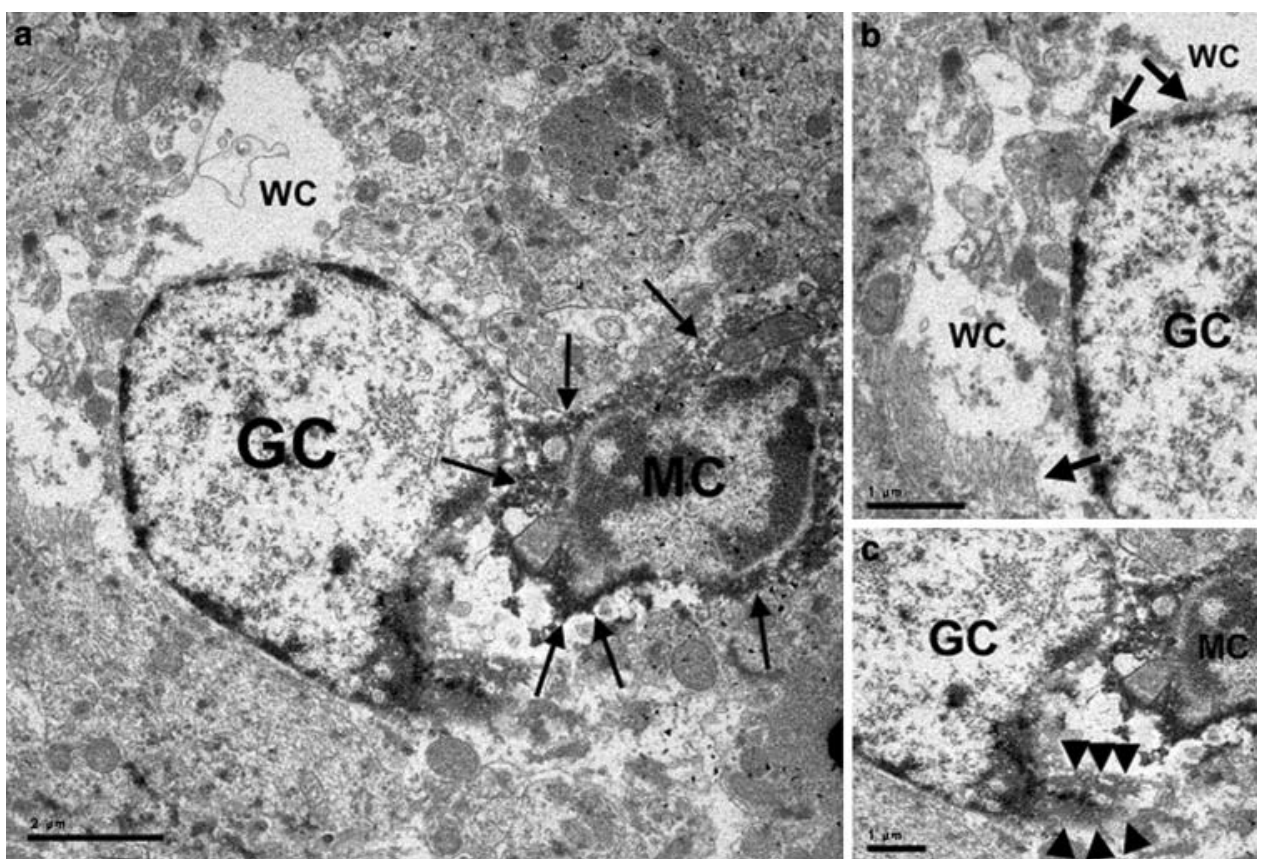

Fig. 8 Electron micrographs of another granule cell body that has an Iba1-labeled microglial cell body within it. a A microglial cell body (MC) with Iba1-immunoreaction product (arrows) in its perikaryal cytoplasm. Note that it is in contact with the nuclear envelope of this granule cell (GC) that displays watery perikaryal cytoplasm (WC). The typically round nucleus of granule cells is not observed for this granule cell's nucleus which is indented. b The watery perikaryal cytoplasm (WC) and collapsed organelles (arrows) are shown for the non-apposed side of the granule cell's nucleus (GC). In c, portions of the nuclear envelope appear separated from the parent nucleus and nuclear pores (arrows) are observed in this region that is apposed by the microglial cell (MC). Scale bars $2 \mu \mathrm{m}$ in $\mathbf{a}$, and $1 \mu \mathrm{m}$ in $\mathbf{b}$ and $\mathbf{c}$ 


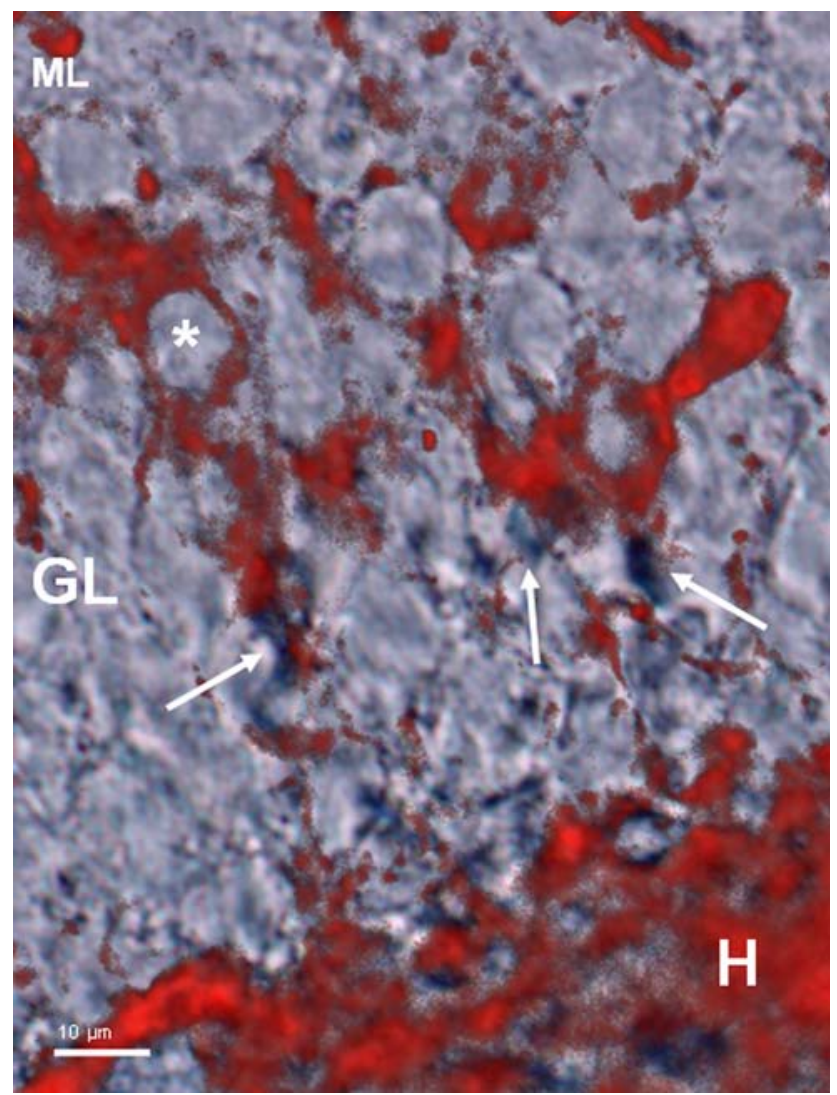

Fig. 9 Iba1-labeled cell bodies and processes associated with damaged granule cells in the granule cell layer (GL). Three trypan bluelabeled cells (white arrows) are found in the GL and indicate a lack of plasma membrane integrity. Notice that they are apposed by the processes and cell bodies of Iba1-labeled cells (red). One unlabeled granule cell (asterisk) in the GL is surrounded by an Iba1-labeled cell body and its processes. Additional trypan blue-labeled cells are found at the GL border with the hilus $(\mathrm{H})$ where a plexus of Iba1-labeled cells and processes are found. A small part of the molecular layer (ML) appears in the top left corner of this panel. Scale bar $10 \mu \mathrm{m}$ dentate gyrus. Although some studies have reported low numbers of apoptotic cells (Ciaroni et al. 2002; Amrein et al. 2004; Kuhn et al. 2005; Ben Abdallah et al. 2007), they were located at the hilar border of the granule cell layer, a region that matches the location of adult neurogenesis. Therefore, these apoptotic cells may be either progenitor cells or newborn neurons that fail to become integrated into hippocampal circuitry. The fact that many of the granule cells involved in microglia-associated granule cell death in the present study are located at the molecular layer border with the granule cell layer suggests that mature granule cells are dying because previous studies have shown that granule cells with somata in this part of the granule cell layer are older than the younger granule cells being born in the adult (Zhao et al. 2006). Furthermore, many of these granule cells targeted by microglial cells have axosomatic synapses on their cell body and newborn neurons usually take more than 4 weeks before axosomatic synapses appear on them (Kaplan and Hinds 1977; Toni et al. 2007). Therefore, the granule cells undergoing microglia-associated granule cell death are probably more mature than newborn neurons.

Microglia-associated granule cell death was also observed at the hilar border of the granule cell layer, indicating that some of the less mature granule cells may die via this pathway. We suggest that this novel pathway of granule cell death at the hilar border occurs to compensate for the excess neurogenesis in the normal adult dentate gyrus.

Microglial cell morphology in the granule cell layer

Microglial cells display a continuum of morphological states, falling within a range of non-activated or resting,

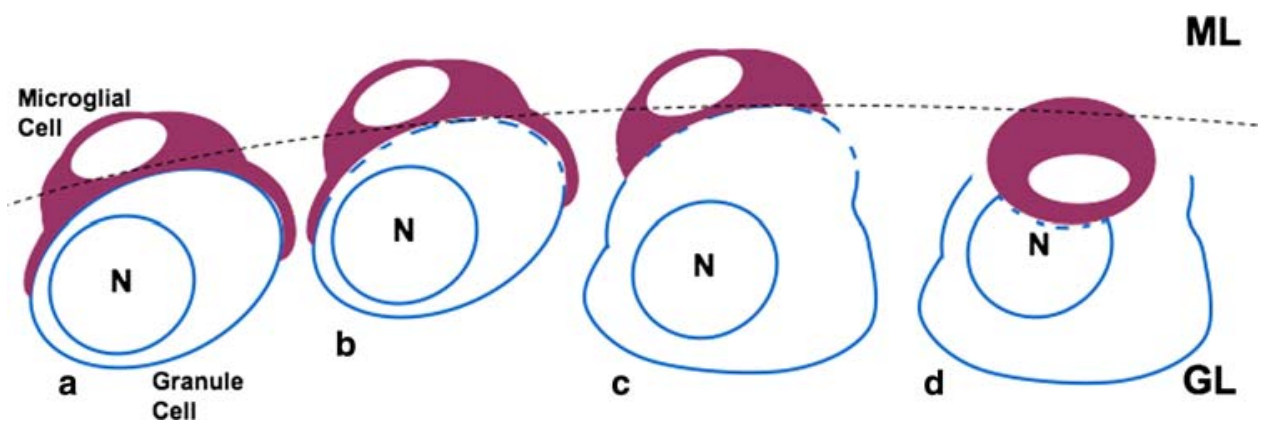

Fig. 10 Schematic diagram of microglia-associated granule cell death showing plasma membrane lysis, neuronal cell body edema, and nuclear phagocytosis. a A microglial cell (purple) and its processes partially surround a single granule cell body (blue) at the molecular layer (ML) border of the granule cell layer (GL). $N$ nucleus of granule cell. b The next stage is represented by openings in the plasma membrane (dashed blue line) of the granule cell body at sites apposed by the microglial cell body and its processes. c An influx of extracellular fluid is hypothesized to occur through the openings in the granule cell's plasma membrane causing hypertrophy of the cell body, a watery perikaryal cytoplasm, damaged intracellular organelles, and a watery nucleoplasm. Note that the microglial cell loses its processes at this stage. $\mathbf{d}$ The microglial cell body lies within the shell of the granule cell body and apposes its nucleus $(N)$, causing its breakdown (dashed blue line) at this stage 
activated and phagocytic states (see review by Biber et al. 2007; Del Rio Hortega, 1932). All previous reports on Iba1-labeled microglial cells in the granule cell layer of the normal adult dentate gyrus described them as being in a resting or non-activated state (Jinno et al. 2007; Olah et al. 2009). Thus, it was truly surprising to observe the pathological changes of granule cells associated with Iba1labeled microglial cells in the present study because the microglial cells involved in this process of "microgliaassociated granule cell death" do not display the typical "activated" morphology. Perhaps, this is one reason why this mechanism of cell death has escaped detection by routine histological and immunocytochemical methods. Another possibility is that this "atypical" mechanism of cell elimination might be necessary to elude an autoimmune response by microglial cells against the healthy, surviving granule cells. Consistent with this notion is the lack of reactive astrocytes around the targeted granule cells undergoing microglia-associated granule cell death. Thus, despite their benign morphology, we suggest that microglial cells play an active role in the death of mature granule cells through a process consisting of four stages (Fig. 10).

It needs to be emphasized that the microglial cells adjacent to and within the granule cell layer have different morphologies. Some of them are multipolar with processes emanating from several poles of their cell body (see cell in Fig. 3 at hilar border), while others display only one or two processes that wrap around a granule cell body (compare cells in Figs. 2, 3, both located at the molecular layer border). In addition, other microglial cells lacked apparent processes and were located within the shell of a granule cell (Figs. 3, 7, 8). The morphology of this latter stage for microglial cells in the present study is similar to the round shape of the Gitter cell (compound granular corpuscle or rounded macrophages) observed at the end-stage of phagocytosis (Del Rio Hortega 1932). We hypothesize that these different morphologies for microglial cells in the granule cell layer represent different stages in the death sequence of its associated/targeted granule cell. Thus, an updated morphological nomenclature might be needed for a more detailed description of microglial cells involved in this novel form of neuronal death. Emerging technology using real-time systems, such as two-photon microscopy, will greatly aid in the temporal sequencing of the events involved with "microglia-associated granule cell death."

\section{Technical considerations}

The granule cells that are apposed by Iba1-labeled microglial cells exhibit unusual morphology at the electron microscopic level. The presence of watery nucleoplasm and perikaryal cytoplasm within the granule cells that had their plasma membranes disrupted appears selective for these granule cells because the neighboring granule cells had normal morphology. In addition, the neuropil adjacent to the dying granule cells exhibited normal characteristics. Therefore, the changes observed in the edematous granule cells at the electron microscopic level probably do not represent artifacts of fixation.

Hippocampal slices for Trypan blue staining are prepared in ice-cold oxygenated ACSF and incubated in ACSF for $1 \mathrm{~h}$ at $20^{\circ} \mathrm{C}$ followed by Trypan blue incubation. We cannot exclude the possibility that hypoxic-ischemic damage due to slice preparation results in the migration/ apposition of microglia to their target granule cells during the incubation period. However, this is unlikely because the slice temperature was not physiological enough for this to occur.

Acknowledgments The authors would like to recognize Dr. Zhiyin Shan for technical expertise and Drs. Edwin S. Monuki, Daniel McCloskey, and Richard T. Robertson for valuable discussions of our data and comments on the manuscript. We also acknowledge support from NIH grant R01-NS38331 (to CER) and R01-AA016100 (to IS).

Open Access This article is distributed under the terms of the Creative Commons Attribution Noncommercial License which permits any noncommercial use, distribution, and reproduction in any medium, provided the original author(s) and source are credited.

\section{References}

Ackman JB, Siddiqi F, Walikonis RS, LoTurco JJ (2006) Fusion of microglia with pyramidal neurons after retroviral infection. J Neurosci 26:11413-11422

Amrein I, Slomianka L, Lipp HP (2004) Granule cell number, cell death and cell proliferation in the dentate gyrus of wild-living rodents. Eur J Neurosci 20:3342-3350

Ben Abdallah NM, Slomianka L, Lipp HP (2007) Reversible effect of $\mathrm{X}$-irradiation on proliferation, neurogenesis, and cell death in the dentate gyrus of adult mice. Hippocampus 17:1230-1240

Biber K, Neumann H, Inoue K, Boddeke HW (2007) Neuronal 'On' and 'Off' signals control microglia. Trends Neurosci 30:596-602

Biebl M, Cooper CM, Winkler J, Kuhn HG (2000) Analysis of neurogenesis and programmed cell death reveals a self-renewing capacity in the adult rat brain. Neurosci Lett 291:17-20

Cameron HA, McKay RD (2001) Adult neurogenesis produces a large pool of new granule cells in the dentate gyrus. J Comp Neurol 435:406-417

Christie BR, Cameron HA (2006) Neurogenesis in the adult hippocampus. Hippocampus 16:199-207

Ciaroni S, Cecchini T, Ferri P, Cuppini R, Ambrogini P, Santi S, Benedetti S, Del Grande P, Papa S (2002) Neural precursor proliferation and newborn cell survival in the adult rat dentate gyrus are affected by vitamin E deficiency. Neurosci Res 44:369-377

Colwell CS, Altemus KL, Cepeda C, Levine MS (1996) Regulation of N-methyl-D-aspartate-induced toxicity in the neostriatum: a role for metabotropic glutamate receptors? Proc Natl Acad Sci USA 93:1200-1204

Dalmau I, Finsen B, Zimmer J, González B, Castellano B (1998) Development of microglia in the postnatal rat hippocampus. Hippocampus 8:458-474 
Davalos D, Grutzendler J, Yang G, Kim JV, Zuo Y, Jung S, Littman DR, Dustin ML, Gan WB (2005) ATP mediates rapid microglial response to local brain injury in vivo. Nat Neurosci 8:752-758

de Bilbao F, Guarin E, Nef P, Vallet P, Giannakopoulos P, DuboisDauphin M (1999) Postnatal distribution of cpp32/caspase 3 mRNA in the mouse central nervous system: an in situ hybridization study. J Comp Neurol 409:339-357

Del Rio Hortega P (1932) Microglia. In: Penfield W (ed) Cytology and cellular pathology of the nervous system, vol 2. Hoeber, New York, pp 483-534

Gehrmann J, Banati RB, Wiessner C, Hossmann KA, Kreutzberg GW (1995) Reactive microglia in cerebral ischaemia: an early mediator of tissue damage? Neuropathol Appl Neurobiol 21:277-289

Hirt UA, Gantner F, Leist M (2000) Phagocytosis of nonapoptotic cells dying by caspase-independent mechanisms. J Immunol 164:6520-6529

Jinno S, Fleischer F, Eckel S, Schmidt V, Kosaka T (2007) Spatial arrangement of microglia in the mouse hippocampus: a stereological study in comparison with astrocytes. Glia 55:1334-1347

Kaplan MS, Hinds JW (1977) Neurogenesis in the adult rat: electron microscopic analysis of light radioautographs. Science 197:1092-1094

Kempermann G, Kuhn HG, Gage FH (1997) Genetic influence on neurogenesis in the dentate gyrus of adult mice. Proc Natl Acad Sci USA 94:10409-10414

Kempermann G, Gast D, Kronenberg G, Yamaguchi M, Gage FH (2003) Early determination and long-term persistence of adultgenerated new neurons in the hippocampus of mice. Development 130:391-399

Kuhn HG, Biebl M, Wilhelm D, Li M, Friedlander RM, Winkler J (2005) Increased generation of granule cells in adult Bcl-2overexpressing mice: a role for cell death during continued hippocampal neurogenesis. Eur J Neurosci 22:1907-1915

Laplagne DA, Kamienkowski JE, Espósito MS, Piatti VC, Zhao C, Gage FH, Schinder AF (2007) Similar GABAergic inputs in dentate granule cells born during embryonic and adult neurogenesis. Eur J Neurosci 25:2973-2981

Moga MM, Dempah D, Zhou D (2005) Annexin 7-immunoreactive microglia in the hippocampus of control and adrenalectomized rats. Neurosci Lett 380:42-47

Nimmerjahn A, Kirchhoff F, Helmchen F (2005) Resting microglial cells are highly dynamic surveillants of brain parenchyma in vivo. Science 308:1314-1318

Olah M, Ping G, De Haas AH, Brouwer N, Meerlo P, Van Der Zee EA, Biber K, Boddeke HW (2009) Enhanced hippocampal neurogenesis in the absence of microglia $\mathrm{T}$ cell interaction and microglia activation in the murine running wheel model. Glia 57:1046-1061
Overstreet-Wadiche LS, Westbrook GL (2006) Functional maturation of adult-generated granule cells. Hippocampus 16:208-215

Portera-Cailliau C, Price DL, Martin LJ (1997) Non-NMDA and NMDA receptor-mediated excitotoxic neuronal deaths in adult brain are morphologically distinct: further evidence for an apoptosis-necrosis continuum. J Comp Neurol 378:88-104

Schmued LC, Hopkins KJ (2000) Fluoro-Jade: novel fluorochromes for detecting toxicant-induced neuronal degeneration. Toxicol Pathol 28:91-99

Shapiro LA, Ribak CE (2006) Newly born dentate granule neurons after pilocarpine-induced epilepsy have hilar basal dendrites with immature synapses. Epilepsy Res 69:53-66

Shapiro LA, Upadhyaya P, Ribak CE (2007) Spatiotemporal profile of dendritic outgrowth from newly born granule cells in the adult rat dentate gyrus. Brain Res 1149:30-37

Shapiro LA, Wang L, Ribak CE (2008) Rapid astrocyte and microglial activation following pilocarpine-induced seizures in rats. Epilepsia 49(Suppl 2):33-41

Shapiro LA, Perez ZD, Foresti ML, Arisi GM, Ribak CE (2009) Morphological and ultrastructural features of Iba1-immunolabeled microglial cells in the hippocampal dentate gyrus. Brain Res 1266:29-36

Sloviter RS, Dean E, Sollas AL, Goodman JH (1996) Apoptosis and necrosis induced in different hippocampal neuron populations by repetitive perforant path stimulation in the rat. J Comp Neurol 366:516-533

Thanos S, Kacza J, Seeger J, Mey J (1994) Old dyes for new scopes: the phagocytosis-dependent long-term fluorescence labelling of microglial cells in vivo. Trends Neurosci 17:177-182

Toni N, Teng EM, Bushong EA, Aimone JB, Zhao C, Consiglio A, van Praag H, Martone ME, Ellisman MH, Gage FH (2007) Synapse formation on neurons born in the adult hippocampus. Nat Neurosci 10:727-734

Toni N, Laplagne DA, Zhao C, Lombardi G, Ribak CE, Gage FH, Schinder AF (2008) Neurons born in the adult dentate gyrus form functional synapses with target cells. Nat Neurosci 11:901907

van Praag H, Schinder AF, Christie BR, Toni N, Palmer TD, Gage FH (2002) Functional neurogenesis in the adult hippocampus. Nature 415:1030-1034

Wirenfeldt M, Dalmau I, Finsen B (2003) Estimation of absolute microglial cell numbers in mouse fascia dentata using unbiased and efficient stereological cell counting principles. Glia 44:129 139

Zhao C, Teng EM, Summers RG Jr, Ming GL, Gage FH (2006) Distinct morphological stages of dentate granule neuron maturation in the adult mouse hippocampus. J Neurosci 26:3-11 